\title{
Droplet sizing in spray flame synthesis using wide-angle light scattering (WALS)
}

\author{
Simon Aßmann ${ }^{1,2,3} \cdot$ Bettina Münsterjohann ${ }^{1,2,3} \cdot$ Franz J. T. Huber $^{1,2,3} \cdot$ Stefan Will ${ }^{1,2,3}$
}

Received: 4 February 2020 / Accepted: 14 April 2020 / Published online: 28 April 2020

(c) The Author(s) 2020

\begin{abstract}
In spray flame synthesis the processes of spray formation and evaporation of the single droplets greatly affect the morphology and size of particles formed. An in situ measurement of these parameters is thus essential for process control and development. In this work, wide-angle light scattering (WALS) is applied to measure droplet sizes in a spray flame. The scattering data of the spherical droplets are evaluated by applying Mie-theory. For droplet sizing, the number of characteristic maxima in the scattering pattern and the measured scattering intensities are evaluated. Droplet size distributions and their parameters were determined by repetitive exposures in various heights above the nozzle outlet for two solvents: pure ethanol and a mixture of ethanol and 2-ethylhexanoic acid at a volume ratio of 35/65. While for ethanol the median droplet size decreases with increasing height, it decreases less for the mixture, which in general exhibits increased droplet sizes for all heights compared to pure ethanol. Furthermore, we could show that using air instead of nitrogen as a co-flow barely affects droplet evaporation in the flame.
\end{abstract}

\section{Introduction}

For a wide range of materials such as fumed silica, titania or indium tin oxide, gas-phase synthesis is a well-established process for the production of nanoparticles on an industrial scale [1-3]. Particle synthesis can be accomplished in a continuous process yielding products with high purity. Among various production processes in the gas phase, spray flame synthesis (SFS), often also termed flame-spray pyrolysis (FSP), is a promising approach for a continuous and costeffective production of a variety of functional semiconductor- and metal-based inorganic nanoparticles like titania, zirconia, iron oxides and many others at industrial scales [4-11]. Non-volatile precursor substances are dissolved in

Stefan Will

stefan.will@fau.de

1 Lehrstuhl für Technische Thermodynamik (LTT), FriedrichAlexander-Universität Erlangen-Nürnberg (FAU), Am Weichselgarten 8, 91058 Erlangen, Germany

2 Erlangen Graduate School in Advanced Optical Technologies (SAOT), Friedrich-Alexander-Universität Erlangen-Nürnberg (FAU), 91052 Erlangen, Germany

3 Cluster of Excellence Engineering of Advanced Materials (EAM), Friedrich-Alexander-Universität Erlangen-Nürnberg (FAU), 91052 Erlangen, Germany a flammable liquid and sprayed into a hot pilot flame. The solvent is rapidly evaporated and burned, and the precursor is chemically converted into the desired product. Complex and high-purity nanomaterials with defined functionality and size distribution can be produced by dedicated combinations of solvents and precursors and a systematic process control. However, a successful industrial scale-up requires a profound understanding of the fundamental processes like spray and droplet formation, evaporation and combustion of the solvent, the chemical reactions and interactions between educts and products and the particle formation mechanisms [12]. Up to now, these processes have not been systematically investigated for the variety of material systems of interest. Besides, many chemicals and solvents used in this process are expensive and require appropriate cost-efficient substitutions. Within the interdisciplinary program on 'Nanoparticle Synthesis in Spray Flames SpraySyn: Measurement, Simulation, Processes' (SPP1980) of the German Research Foundation (DFG) a standardized burner system for spray flame synthesis-the SpraySyn burnerwas designed allowing for a comprehensive and reproducible examination of the fundamentals of the SFS under standardized conditions (www.spraysyn.org). Performing a flame benchmarking procedure the standardized operation of the SpraySyn burner at all participating institutions is ensured $[13,14]$. 
The investigation of the complex SFS processes like spray and particle formation is feasible with a variety of laserbased in situ measurement techniques that can yield results on process properties with high temporal resolution and without disturbing the measurement object. Laser Doppler anemometry (LDA) and phase Doppler anemometry (PDA) in particular are widely used in fluid dynamic research and industrial applications for spray-characterization, as these methods allow to measure velocity, size and concentration of spherical objects like droplets and bubbles simultaneously at a high repetition rate [15-20]. In the dual-beam approach, a widely used configuration, two coherent incident laser beams cross at an intersection angle and form an interference fringe pattern. Moving objects crossing that interference pattern scatter light elastically, which can be temporally resolved by fast photodetectors. The velocity of the object can then be determined from the frequency of the detected scattered light. Detecting the signal with multiple detectors, the diameter of the scattering object can be related to the phase difference between the photodetectors. Consequently, PDA devices are capable of measuring size and velocity simultaneously [21-26]. However, PDA requires sufficiently low droplet concentrations as problems arise if several droplets are simultaneously present in the measurement volume.

Classical elastic light scattering (ELS) techniques detecting the angular distribution of scattered light under multiple angles are favorably used for the characterization of microand especially nano-sized particles regarding size and shape [27]. ELS has been successfully applied to 2D-investigations of particle formation in laminar diffusion flames and premixed flames [28, 29]. However, applying 2D light scattering techniques to turbulent systems like the SFS would require a fast detection system that allows for a synchronous imaging of scattered light from multiple scattering angles. To overcome this drawback, the wide-angle light scattering approach was developed by Oltmann et al. [30] and applied to measure aggregation in turbulent flames [31]. Elastically scattered light is collected by an ellipsoidal mirror and imaged onto a CCD-camera over a wide angular range and with a high angular resolution on a single-shot basis. Furthermore, this approach allows to derive the underlying particle size distribution by inverse analysis of the acquired scattering data due to the high angular resolution $[32,33]$.

Although the WALS-approach has only been applied on the investigation of nanoparticles of different morphology so far, it can generally be used to capture scattering signals of any scattering objects within the measurement volume, regardless of size and shape. Applying the WALS-approach to the SFS process not only allows for a morphological characterization of the produced nanoparticles, but also for an investigation of the solvent spray from which the particles are synthesized. The atomization of the solvent leads to the formation of droplets of different sizes and distributions along the radial and axial extent of the spray flame. Their scattering behavior can be described by the classical Mietheory [34-36]. Since smaller droplets evaporate much faster than large droplets, a transition zone is formed in which droplets and first particles are present simultaneously. Thus, approaching the spray region of the SFS with decreasing height above burner, the scattering signal of particles is superimposed by the signal of dispersed solvent droplets. As the WALS-approach is capable of acquiring scattering signals with a high angular resolution, an investigation of such processes becomes feasible with the goal of a simultaneous determination of particle and droplet sizes in regions where both fractions are present.

In this work, we present the first application of the WALS technique for the determination of droplet sizes and their distributions in a spray flame. Measurements are performed on the SpraySyn burner at different heights above the burner surface. The spray flame was operated with pure ethanol and a mixture of $35 \%$ vol. ethanol and $65 \%$ vol. ethylhexanoic acid as solvents [14]. Both solvents were used without precursor substances to avoid particle formation and the superposition of scattering signals from droplets and particles for these first investigations. We explain in detail the derivation of the droplet size and their distribution from the scattering data and discuss current limitations of the WALS-approach for droplet sizing.

\section{Theory}

The behavior of elastic light scattering from spherical objects can be described by Mie theory [34]. Good overviews for Mie scattering calculations can be found in in the literature [35-38]. For our investigations of droplet sizes in a spray flame with the WALS approach, we use a Matlab based Mie scattering algorithm for spheres based on Bohren and Hufmann [36]. Also Wiscombe [38] provided a very good description of the Mie formalism relevant for the computation of Mie scattering signals. Here, we only give a short overview of the equations relevant to calculate scattering signals for homogeneous spheres of arbitrary diameter $d$ following [36] and [38].

The radiant intensity of scattered light $I$ is dependent on its polarization. In this work, only vertically polarized laser light is used for illumination. The vertical component $I_{\perp}$ (measured in the conducted experiments) can be calculated from the scattering amplitude $S$ [36]:

$I_{\perp}=|S|^{2}=\left|\sum_{n=1}^{N} \frac{2 n+1}{n(n+1)}\left(a_{n} \pi_{n}+b_{n} \tau_{n}\right)\right|^{2}$. 
Assuming equal permeabilities of particle and surrounding medium, the Mie scattering coefficients $a_{n}$ and $b_{n}$ are

$a_{n}=\frac{\left[D_{n}(m x) / m+n / x\right] \psi_{n}(x)-\psi_{n-1}(x)}{\left[D_{n}(m x) / m+n / x\right] \xi_{n}(x)-\xi_{n-1}(x)}$,

$b_{n}=\frac{\left[m D_{n}(m x)+n / x\right] \psi_{n}(x)-\psi_{n-1}(x)}{\left[m D_{n}(m x)+n / x\right] \xi_{n}(x)-\xi_{n-1}(x)}$.

here, $D_{n}(m x)$ is the logarithmic derivative of $\psi_{n}(m x)$ with $D_{n}(m x)=\mathrm{d} / \mathrm{d}(m x) \ln \psi_{n}(m x), m=N_{1} / N_{0}$ is the relative refractive index of the sphere $N_{1}$ to the surrounding medium $N_{0}$ and $x=\pi d / \lambda$ is the size parameter, whereby $\lambda$ is the wavelength of the incident light. The values for the RiccatiBessel functions $\psi_{n}(x)$ and $\xi_{n}(x)$ are calculated by a series expansion with termination criteria of $n \leq N$ with

$N=x+4 x^{\frac{1}{3}}+2$,

following the results of Wiscombe [38]. The functions $\pi_{n}$ and $\tau_{n}$ are dependent on the scattering angle $\theta$ with

$\pi_{n}=\frac{2 n-1}{n-1} \cos \theta \pi_{n-1}-\frac{n}{n-1} \pi_{n-2}$,

$\tau_{n}=n \cos \theta \pi_{n}-(n+1) \pi_{n-1}$,

and have to be computed by upward recurrence up to $n \leq N$ with $\pi_{0}=0$ and $\pi_{1}=1$.

\section{Experimental setup}

A detailed description of the burner configuration can be found in the work of Schneider et al. [14]. Here, only a short overview of the burner system operation, the volume and mass flows of the used gases and solvents for the pilot and spray flame are given. A cross-section schematic of the SpraySyn burner can be found in Fig. 1. The central element of the burner is a two-fluid nozzle where the liquid solvent is injected into a pilot flame and atomized by a dispersion gas to form the spray flame. The spray flame is shielded from environmental disturbances by a coflow of inert gas. A homogeneous distribution of the supply gases for both the pilot flame and the coflow are ensured by a porous bronze sinter matrix around the central nozzle. A graphite sealing separates both supply flows. The pilot flame is fed by a lean mixture of 2.0 standard liters per minute ( $\mathrm{slm}$ ) methane (purity 2.5) and $16.0 \mathrm{slm}$ oxygen $\left(\mathrm{O}_{2}\right.$, purity 2.5$)$, the coflow by nitrogen $\left(\mathrm{N}_{2}\right.$, purity 5.0$)$ or pressurized and dried air with a flow rate of $120 \mathrm{slm}$. As dispersion gas $\mathrm{O}_{2}$ is employed with a flow rate of $10.0 \mathrm{slm}$. The solvent is added continuously at a flow rate of $2.0 \mathrm{ml} / \mathrm{min}$ with a twin syringe pump.

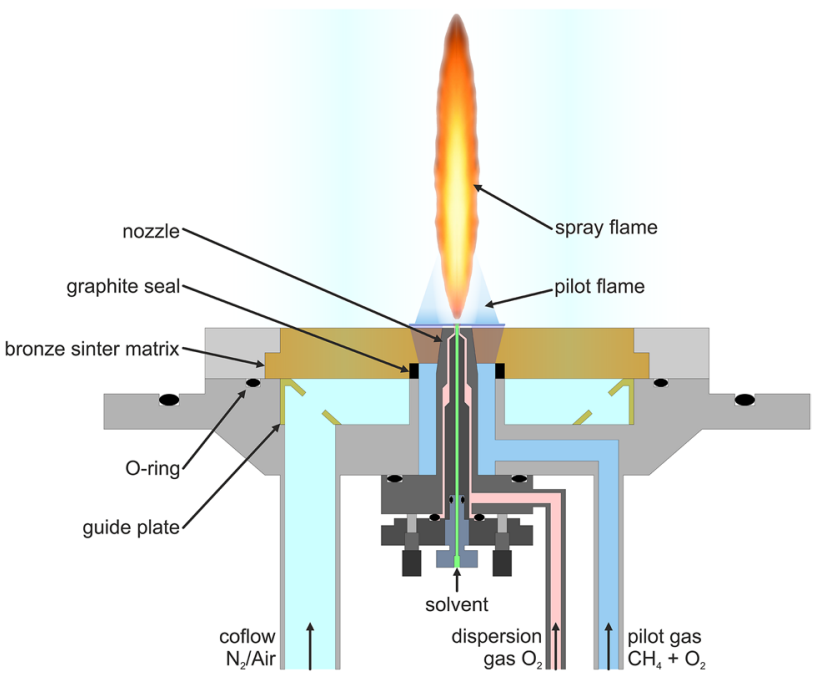

Fig. 1 Schematics of the SpraySyn burner following [14]

For the flame benchmarking pure ethanol (purity $\geq 99.5 \%$ ) is used. For spray formation, pure ethanol and a mixture of ethanol and ethylhexanoic acid (EHA, purity $\geq 99.0 \%$ ) at a volume ratio of 35:65 are utilized. The correct operation of the burner system and the reproducibility of the flame was ensured by applying the benchmarking procedure described by Schneider et al. [14].

Figure 2 shows a scheme of the optical setup used, which is based on those of Oltmann et al [31] and of Huber et al. [39] for nanoparticle measurements with WALS. A pulsed Nd:YAG laser (Quantel, Q-Smart 450) operated at $532 \mathrm{~nm}$ with a maximum energy of $190 \mathrm{~mJ}$ per pulse, a pulse width of $5 \mathrm{~ns}$ and a repetition rate of $10 \mathrm{~Hz}$ is used as an incident light source. A combination of a half-wave plate and a thin-film polarizer is employed for the regulation of the pulse energy to less than $30 \mu \mathrm{J}$ in the center area of the measurement volume to avoid oversaturation of the camera. An aperture with an inner diameter of $1.0 \mathrm{~mm}$ placed in the beam path is imaged into the measurement volume by a $4 f$-imaging setup consisting of two spherical planoconvex lenses with an identical focal length of $f=300 \mathrm{~mm}$. The distance between both lenses is $2 f$, the distance between aperture and first lens as well as between the second lens and the measurement volume is $f$. This setup allows for a parallel beam and a sharp top hat laser profile with a diameter of $1 \mathrm{~mm}$ within the measurement volume. A half-wave plate in combination with a Glan-laser-polarizer (extinction ratio 100,000:1) guarantees vertically polarized laser light with a high purity. Scattering signals of droplets in the measurement volume are collected with an ellipsoidal WALS-mirror in the range between $10^{\circ}$ and $170^{\circ}$ alike the one used by Oltmann et al. [30] with a focal length of $\Delta f=600 \mathrm{~mm}$ and a diameter at the first focal point (and the measurement volume) of $D=250 \mathrm{~mm}$. Two $10 \mathrm{~mm}$ slits on each side of the 


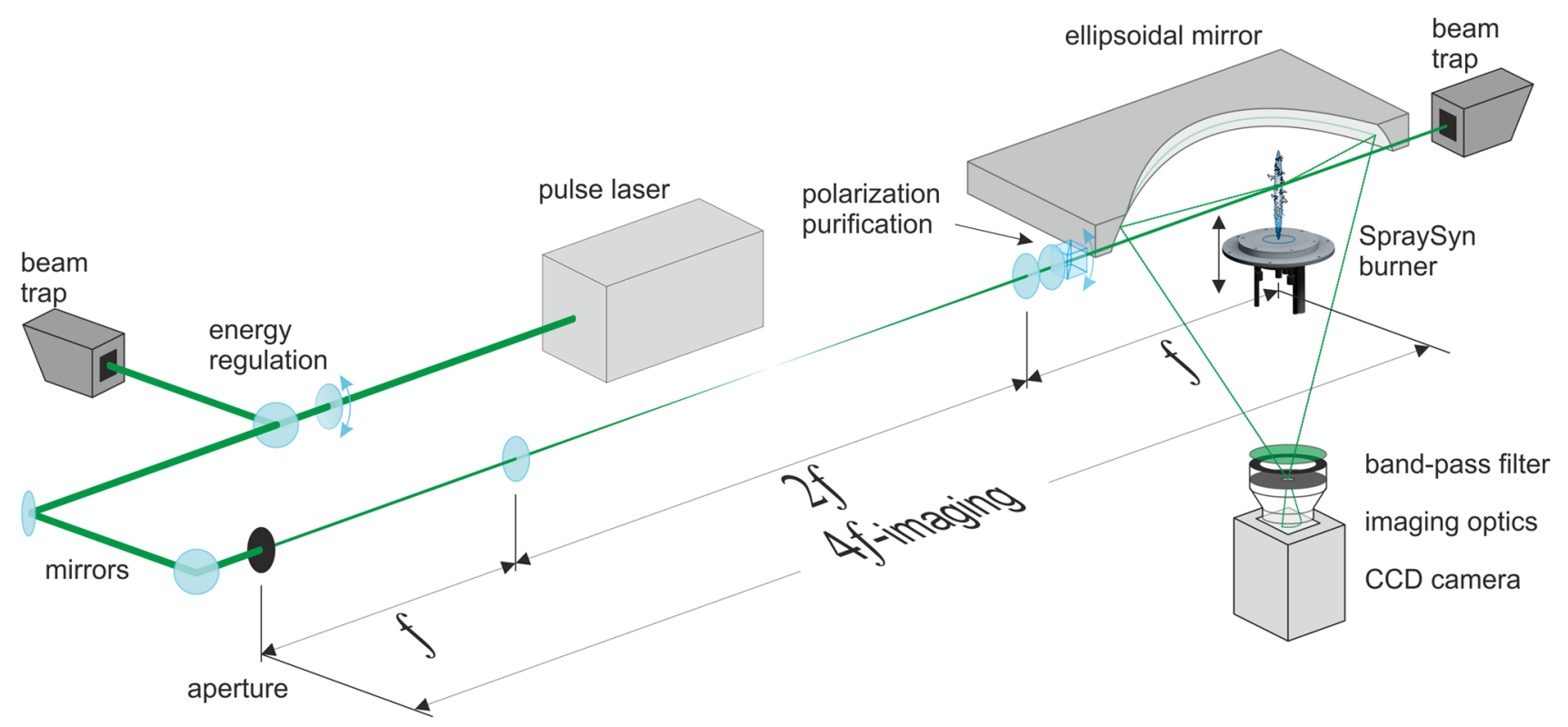

Fig. 2 Schematic of the optical WALS setup for droplet measurements (for a better illustration only one half of the mirror is shown)

mirror allow the laser beam to pass through the measurement volume. The mirrored surface is coated with amorphous nickel exhibiting a surface roughness of less than $10 \mathrm{~nm}$ and an accuracy of better than $2 \mu \mathrm{m}$. The mirror reflects the scattered light and focusses it into the second focal point, where only elastically scattered light is filtered with a bandpass filter (center wavelength $535 \mathrm{~nm}$ ) and imaged onto the sensor of a monochromatic CCD-camera (Allied Vision Technologies GmbH, Pike F-100B, $1000 \times 1000$ pixel, 16 bit) by a camera lens with a focal length of $f=12.5 \mathrm{~mm}$. The laser beam, the ellipsoidal mirror and the position of the camera have been aligned precisely to achieve symmetrical scattering images.

Droplets passing the measurement volume dislocated from its center (the first focal point of the mirror) affect the detection of their elastically scattered light onto the mirror sections as it can be seen in Fig. 3. The measurement region (red area) for a specific detection angle is the cross-section of the laser region (green area) and the field of view of the detector (blue area). Though a single camera is used for imaging the mirror surface and the scattered light within, we can simplify the illustration by assuming multiple single cameras at different angular positions around the mirror surface (grey area). The size of the measurement region is angle-dependent following a $1 / \sin \theta$-dependency [30, 39]. WALS data of nanoparticle ensembles filling the measurement region at any detection angle, therefore, require an appropriate calibration using isotropic scattering from gases like nitrogen. In contrast, as in this work only scattering signals from a single droplet (black circle) within the measurement region are evaluated, no such calibration is needed. However, within a spray, droplets may pass the measurement region at different positions. For a perfectly centered droplet, the scattered light (full green lines) is collected at all scattering angles and symmetrically for left and right mirror half (Fig. 3, a). With increasing distance of the droplet to the scattering center, the detection of scattered light shifts towards lower and larger angles and also shifts between the mirror sections (Fig. 3, b). Depending on the position of the scattering droplet within the measurement region, scattered light is blocked by the aperture of the camera and thus not imaged onto the CCD chip (dashed green lines). The extent of the measurement volume from which scattered light can be detected by the camera is strongly dependent on the aperture of the camera (Fig. 3, c). For an $f$-number of $f / 2.0$ the length of the resulting measurement volume is $1.3 \mathrm{~mm}$ under a scattering angle of $90^{\circ}$, for $f / 5.6$ the length is reduced to $0.4 \mathrm{~mm}$, while the other dimensions of the measurement volume are determined by the diameter of the laser beam $(1.0 \mathrm{~mm})$. With decreasing aperture size, the measurement volume is reduced affecting the measured droplet sizes regarding their detectable amount and their size distribution.

We investigated heights above the burner surface (HAB) between $20 \mathrm{~mm}$ and $100 \mathrm{~mm}$ with $10 \mathrm{~mm}$ increments and additionally at $120 \mathrm{~mm}$ using the standard ethanol/EHAmixture and pure ethanol as solvents. Generally, we used air instead of $\mathrm{N}_{2}$ for the coflow, yet, at $40 \mathrm{~mm}, 60 \mathrm{~mm}$ and $80 \mathrm{~mm} \mathrm{HAB}$ we performed measurements on the ethanol spray using $\mathrm{N}_{2}$ as coflow gas additionally to examine a possible influence of the oxygen in the air on droplet formation and evaporation. The burner was adjusted by a movable 


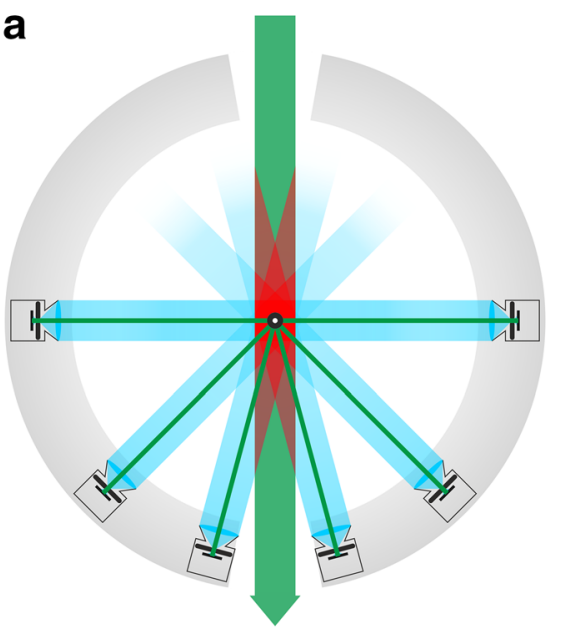

detector with lense, aperture and image sensor

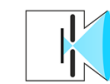

b

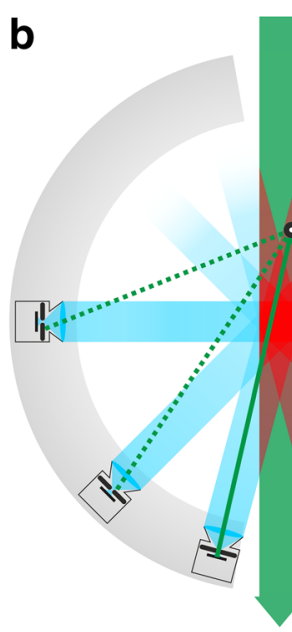
measurement region

Fig. 3 Schematics of wide-angle light scattering from spherical objects depending on the position of scattering object within the measurement region (red areas), field of view of the camera (blue areas) and laser region (green areas). a perfectly centered; $\mathbf{b}$ decen-

stage with a positioning precision of $<0.5 \mathrm{~mm}$. Depending on the observed HAB and solvent, between 2000 and 6000 single scattering images of droplets were acquired. The extent of the measurement volume is determined by the aperture of the camera and generally set to an $f$-number of $f / 2$. Approaching the nozzle of the burner (towards smaller HAB) we observed an increased spray density and thus reduced the size of the measurement volume by decreasing the size of the aperture to $f$-numbers of $f / 4$ for $\mathrm{HAB}<60 \mathrm{~mm}$ and $f / 5.6$ for $\mathrm{HAB}<40 \mathrm{~mm}$. To account for flame luminosity, ambient light and laser reflections as well as thermal noise of the camera, 50 background images were recorded and averaged. All images were taken at an acquisition rate of $5 \mathrm{~Hz}$ and an exposure time of the camera of $50 \mu$ s for an optimized signal-to-noise ratio.

\section{Data evaluation}

To determine droplet sizes and their distributions from measured data on basis of a reference database, certain assumptions for the applicability of the Mie theory must be made first. The calculated signal is not only dependent on the size of the droplets and the scattering angle, but also the refractive indices of the droplet medium and the surrounding atmosphere. Within recent years, a various number of algorithms for the computation of scattering patterns have been developed for different cases, including scattering from particles in absorbing media as well as absorbing,

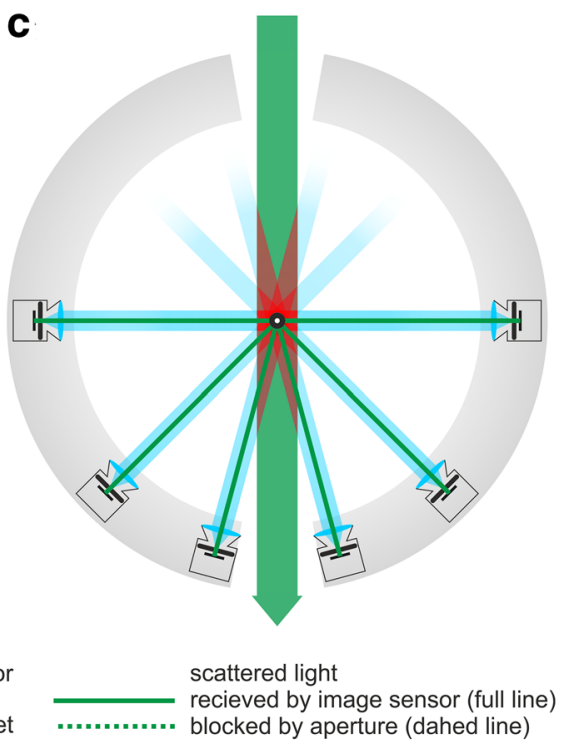

tered from mirror center and along laser axis; c perfectly centered, field of view reduced by higher $f$-number of the aperture of the camera (illustration not true to scale)

spherical multi-layered and ellipsoidal particles [40-48]. In fact, droplets may differ from perfect spherical shape mainly due to shearing forces at the nozzle but also turbulence or the evaporation process occurring in spray flame synthesis. Assuming them aspherical will lead to further unknown parameters, e.g., the shape of the object and its azimuthal and polar angle of orientation, increasing the ill-posedness of the problem. In this work, we assume all droplets of the spray flame to be spherical, allowing for a robust evaluation procedure based on classical Mie theory. Furthermore, we assume constant refractive indices for both the droplets' media and their surrounding gaseous atmosphere, neglecting temperature and concentration-dependent variations of the refractive index induced by heat and mass transfer related mechanisms that might occur during the evaporation process. Some of these simplifications may lead to errors in the derivation of the droplet size, which will be discussed in Sect. 5. Based on these assumptions, suitable parameters, boundaries and increments for the database generation are derived (see Table 1).

All calculations have been performed for vertically polarized light with a wavelength of $532 \mathrm{~nm}$. The minimum angular resolution of the calculation is based on the minimum angular resolution of the experimental setup, which is dependent on the position and resolution of the CCD camera sensor. Here, the average resolution for all pixels in the mirror section of the image is around $0.2^{\circ}$. Thus, data are first calculated with a high angular resolution of $0.01^{\circ}$ and then section-wise averaged to an increment of $0.2^{\circ}$ to account for 
Table 1 Overview of relevant database parameters

\begin{tabular}{lllll}
\hline Variable & Parameter & & Value & Increment \\
\hline$\lambda$ & Incident wavelength & & $532 \mathrm{~nm}$ & Constant \\
$m$ & Complex refractive index & Ethanol & $1.364^{\mathrm{a}}$ & Constant \\
& & Mixture EHA/ethanol & $1.405^{\mathrm{b}}$ & Constant \\
& & Ambient atmosphere & $1.000^{\mathrm{c}}$ & Constant \\
$\theta$ & Scattering angle & & $10^{\circ}-60^{\circ}$ & $0.01^{\circ}$, averaged to $0.2^{\circ}$ \\
$d$ & Sphere size & & $1-60 \mu \mathrm{m}$ & $\sim 1500$ in logarithmic steps \\
\hline
\end{tabular}

${ }^{\mathrm{a}}[49]^{\mathrm{b}}[25]^{\mathrm{c}}[50]$ the averaged scattering light that is imaged onto one camera pixel. Scattering data for estimated droplet sizes in the range of 1-60 $\mu \mathrm{m}$ cover signal intensities over 7 orders of magnitude in the full azimuthal angular range of the measurement system $\left(10^{\circ}-170^{\circ}\right)$, which cannot be resolved completely by the dynamics of the camera system. Thus, we have chosen an evaluation region between $10^{\circ}-60^{\circ}$ as it decreases the intensity dynamic down to approximately four orders of magnitude. Besides, scattering in forward direction is much brighter than in backward direction and also shows periodically occurring local maxima that are characteristic for specific droplet sizes and will be relevant for the evaluation procedure.

The lower boundary for calculation of sphere diameters is set to $1 \mu \mathrm{m}$, which is mostly determined by the expected transition regime from microdroplets to nanoparticles and also the detectable amount of local maxima within the chosen azimuthal region. The larger the spheres, the more local maxima occur in the angular region of interest following a linear behavior. As the signal appears to be frequencylike, we have chosen an approach comparable to calculating the Nyquist frequency for a good estimation of the upper boundary. Between scattering angles of $10^{\circ}$ and $60^{\circ}, 251$ sampling points (sp) are observed resulting in $5.0 \mathrm{sp} /{ }^{\circ}$. The Nyquist frequency is half of the sampling rate. At a spheresize of around $60 \mu \mathrm{m}$, the minimum distance between two peaks for each simulated Mie signal within the chosen azimuthal region is less than the determined Nyquist frequency of $2.5 \mathrm{sp} /{ }^{\circ}$. To minimize the size of the database and evaluation errors arising from a too roughly discretized database, we investigated the deviations between randomly chosen and recovered droplet sizes for different increment types of the database. A database with approximately 1500 calculated sphere sizes between $1 \mu \mathrm{m}$ and $60 \mu \mathrm{m}$ equally distributed in log-space reduces the maximum deviation to below $0.5 \%$.

The droplet diameters are derived from scattering data using a least-square minimization method based on the rootsum-of-squares (RSSQ), which is depicted in Fig. 4 for one mirror section exemplarily. The scattering data are derived from each scattering image and per mirror section following the procedure of Oltmann et al. [30] and Huber et al. [39]. The raw scattering images are background corrected and divided into angular sectors of $0.2^{\circ}$ increment and a radial extent of 15 pixel around the centerline of the mirror (see
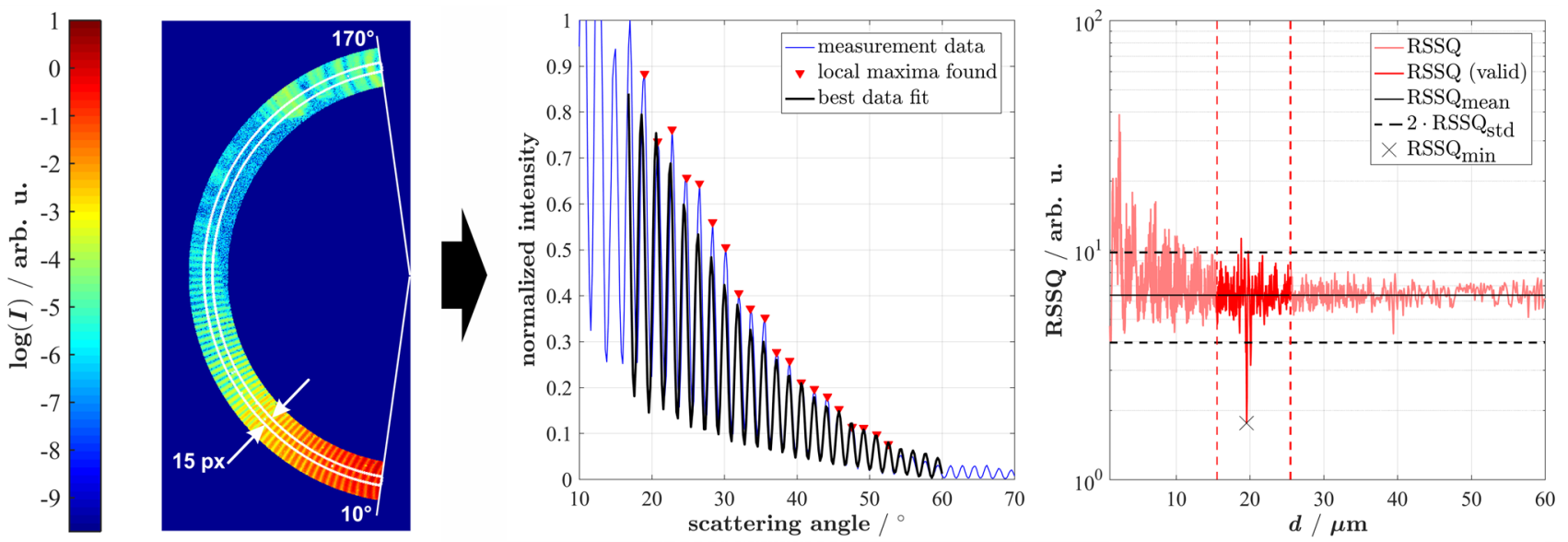

Fig. 4 Example derivation of sphere size from Mie scattering data. Left image: masked scattering image of left mirror section with logarithmic intensities in false colors; middle image: scattering data derived from scattering image, its local maxima within the evaluation region and best data fit derived from least-square minimization; right figure: root-sum-of-squares (RSSQ) of the residuals between measured and calculated signals with minimum RSSQ value for best fit 
Fig. 4, left image). The mean value is derived from all pixels within one angular segment, which typically contains 10-20 pixels, slightly depending on the detection angle. A narrow radial extent of the angular sectors is necessary, as the increased curvature of the scattering pattern towards small (and large) scattering angles would lead to blurred scattering data if image data over a wide radial extent is used for averaging.

A profound and objective data filtering is required to sort out evaluable Mie scattering data and reliably determine droplet sizes of the pyrolysis spray and their distribution along with the height above burner surface. First, data with a maximum intensity level of less than $25 \%$ of the maximum camera output level are rejected. Second, only data with continuous scattering data over an angular extent of at least $10^{\circ}$ in the chosen azimuthal region of $10^{\circ}-60^{\circ}$ are accepted. Hereby, only scattering data between $95 \%$ of the maximum camera output level and the background level are counted. Between $1 \%$ and $98 \%$ camera output level the average deviations from linearity are smaller than $2 \%$ [51]. The background level for scattering data of each mirror half is determined between the scattering angles of $90^{\circ}-100^{\circ}$, as the vertically polarized Mie scattering data show a characteristic intensity minimum in this region for all sphere sizes between $1 \mu \mathrm{m}$ and $60 \mu \mathrm{m}$. Even for large droplets and thus partially oversaturated data at low scattering angles, the camera output in the angular region of $90^{\circ}-100^{\circ}$ remained close to background level of the camera for all scattering images taken in this work. The data are then normalized to its maximum within the valid angular range (see Fig. 4, middle diagram).

The following evaluation steps are applied to data passing both previous filters successfully and are based on the number of local maxima of the scattering data and a leastsquare minimization, which yields the corresponding sphere size of the scattering data. The number of local maxima of the scattering data strongly correlates with the sphere size and thus serves as a first indication in which region of the database the best estimate for the droplet size might be. The Matlab function findpeaks detects the amplitude, position and prominence of the local maxima in the measured and the calculated scattering data for all sphere sizes of the database within the valid angular range. The prominence indicates how outstanding a peak is due to its height and relative position to neighboring peaks. To avoid signal amplitudes from noisy data to be counted as valid local maxima, the minimum prominence was set to $5 \%$ of the maximum intensity level of the scattering data. As it can be seen from the example depicted in Fig. 4, nearly all local maxima are detected very precisely up to a scattering angle of around $53^{\circ}$. Although further local maxima are apparent in the scattering signal beyond $53^{\circ}$, they are not recognized due to the minimum peak prominence. In such cases, the valid angular evaluation range is adjusted accordingly to the maximum angle at which a local maximum is still detected. In addition, scattering data for which no local maximum can be found are not considered further in the evaluation. A first estimate of the sphere size $d$ is then derived by comparing the number of local maxima found in the measured scattering data with those derived from the database.

Within the interval $d \pm 5 \mu \mathrm{m}$ the RSSQ between measured and calculated data for each sphere size is derived (see Fig. 4, right diagram). As both measured and calculated scattering data are normalized by their individual maximum values, a normalizing variable $C$ is used to adjust both signals in absolute intensity for optimized RSSQ calculations. Within the interval $d \pm 5 \mu \mathrm{m}$, the geometric mean value and geometric standard deviation for all RSSQ values are calculated. The RSSQ minimum value determines the most likely sphere size $d$ fitting the scattering data (black cross in the right diagram of Fig. 4) and is valid if it is outside the interval of two geometric standard deviations of the RSSQvalues. However, some residuals may remain due to noise or overlaying scattering from multiple droplets. Depending on the observation height above the burner surface it may occur that, e.g., two droplets are passing the measurement volume in a way that each mirror half collects the scattered light of one of the droplets (c.f. signal paths in Fig. 3). If the deviation of the sphere sizes derived from both mirror sections is larger than $25 \%$, we assume that - at least-scattering data from two droplets has been recorded and thus the results from both mirror sections are accepted individually.

\section{Results and discussion}

In the following, the results for the different burner operations regarding the solvent (ethanol, ethanol/EHA) and variations in the optical set-up are presented. For a first demonstration of the measurement system and evaluation strategy, pure ethanol was used without a precursor. Figure 5 shows the results for the droplet size distribution at two different HABs (30 mm, $80 \mathrm{~mm}$ ); these heights have been chosen, as characteristic features of the evaluation can be well explained. Additionally, reference data from PDA [25] are available for $\mathrm{HAB}=30 \mathrm{~mm}$. At $\mathrm{HAB}=30 \mathrm{~mm}$ the distribution after evaluation of 1995 camera frames shows a wide distribution of droplet diameters $d$ ranging from about $2 \mu \mathrm{m}$ up to $40 \mu \mathrm{m}$, with a median of $13.8 \mu \mathrm{m}$. At this height, 1356 droplet diameters were detected in 1153 evaluable images, corresponding to $58.8 \%$ of all frames. As multiple droplet scattering cannot be avoided at small HABs, a manual verification of all fit results was performed resulting in a median value of $17.3 \mu \mathrm{m}$, i.e. higher by $25 \%$, from 584 evaluable sphere sizes within 562 images (28.2\% of all frames). Data sets in which the best fits do not match the measured 


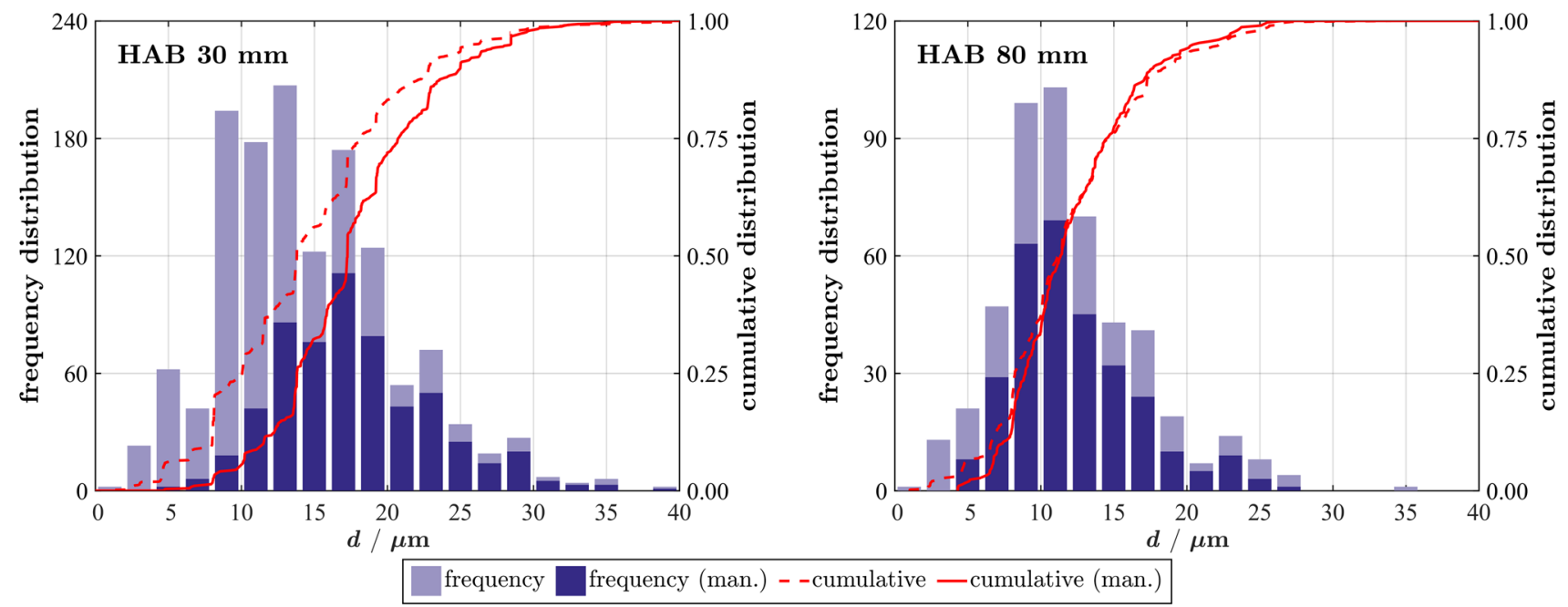

Fig. 5 Frequency and cumulative distributions of ethanol droplets at two different HAB, comparison of distributions derived from automated data filtering and manual verification afterwards (man.); coflow gas: air, $f$-number of camera: $f / 2.0$

data are sorted out during the manual verification of the computer-based evaluation and lead to deviations between the median values. As an example, data that are obviously originating from double droplet scattering may exhibit two major frequencies and thus two distinct RSSQ minima in the computer-based evaluation. However, the fitting algorithm only matches with one of them, fulfilling the filtering criteria described in Sect. 4. The remaining residuals, frequency shaped, are an indication of such artefacts; those data points are thus excluded. In general, scattering of multiple droplets within the measurement volume results in scattering data significantly differing from those ones of a single droplet: either individual local maxima are hardly distinguishable or they do not match maxima of a single droplet. These data are often misinterpreted as scattering from small single droplets from the computer-based evaluation routine leading to an underestimation of the droplet size. Thus, a large fraction of these apparently "small droplet sizes" is filtered out in the manual verification. At $\mathrm{HAB}=80 \mathrm{~mm}, 491$ droplet sizes were determined from $19.6 \%$ of 2492 images with only computer-based evaluation-after manual verification 298 droplets remained in $12.5 \%$ of all images. Both distributions from computer-based evaluation and manual verification are quite congruent yielding median sizes of $11.2 \mu \mathrm{m}$ and $11.4 \mu \mathrm{m}$ in the range of 2-30 $\mu \mathrm{m}$, respectively. Evaporation processes lead to a smaller number concentration of droplets with a much smaller size distribution at increasing HAB. Though in general less scattering data are recorded with increasing $\mathrm{HAB}$, the scattered data themselves are mostly originating from single droplets leading to a more robust and reliable computer-based evaluation.

The cumulative distributions for all investigated HABs derived from computer-based evaluation and after manual verification, respectively, are depicted in Fig. 6. The median values of the distributions derived from computer-based evaluation are in the range of 9.6-14.9 $\mu \mathrm{m}$. After manual verification, the distributions exhibit a quite smooth and similar shape for HABs $>40 \mathrm{~mm}$ with median values between $9.9 \mu \mathrm{m}$ and $19.3 \mu \mathrm{m}$. The droplet distribution at $20 \mathrm{~mm} \mathrm{HAB}$ obtained with computer-based evaluation also exhibits a significant amount of larger droplet sizes $>30 \mu \mathrm{m}$ compared to that derived from manual verification. The superposition of scattering data from several larger droplets leads to a smoother shape of the effective scattering data. However, for some data sets the local maxima of the scattering patterns from each droplet can still be identified in the superimposed scattering data and are then misinterpreted as one larger droplet by the computer-based evaluation.

Figure 7 (left) shows a comparison of various equivalent diameters of the droplet size distributions, including the arithmetic mean $\bar{d}$, the geometric mean $\bar{d}_{\mathrm{g}}$ and the median $\tilde{d}$ as well as the Sauter mean diameter defined as $\bar{d}_{32}=\sum_{i} d_{i}^{3} / \sum_{i} d_{i}^{2}$. As one can see, all equivalent diameters follow the same tendency of decreasing size with increasing HAB. The Sauter mean diameter, with a higher weight on large droplet sizes, is of course shifted to higher values. From the match of geometric mean and median, the assumption of log-normally distributed droplet diameters can be derived in agreement with the shape of the histograms in Fig. 5. Figure 7 (right) depicts the influence of the coflow gas and the $f$-number of the camera aperture on the median of the droplet size distribution. Using nitrogen as coflow gas (black diamonds) instead of air shows no significant influence on the development of the droplet size. Using an $f / 2.0$ aperture with pure ethanol (large blue diamonds), the 

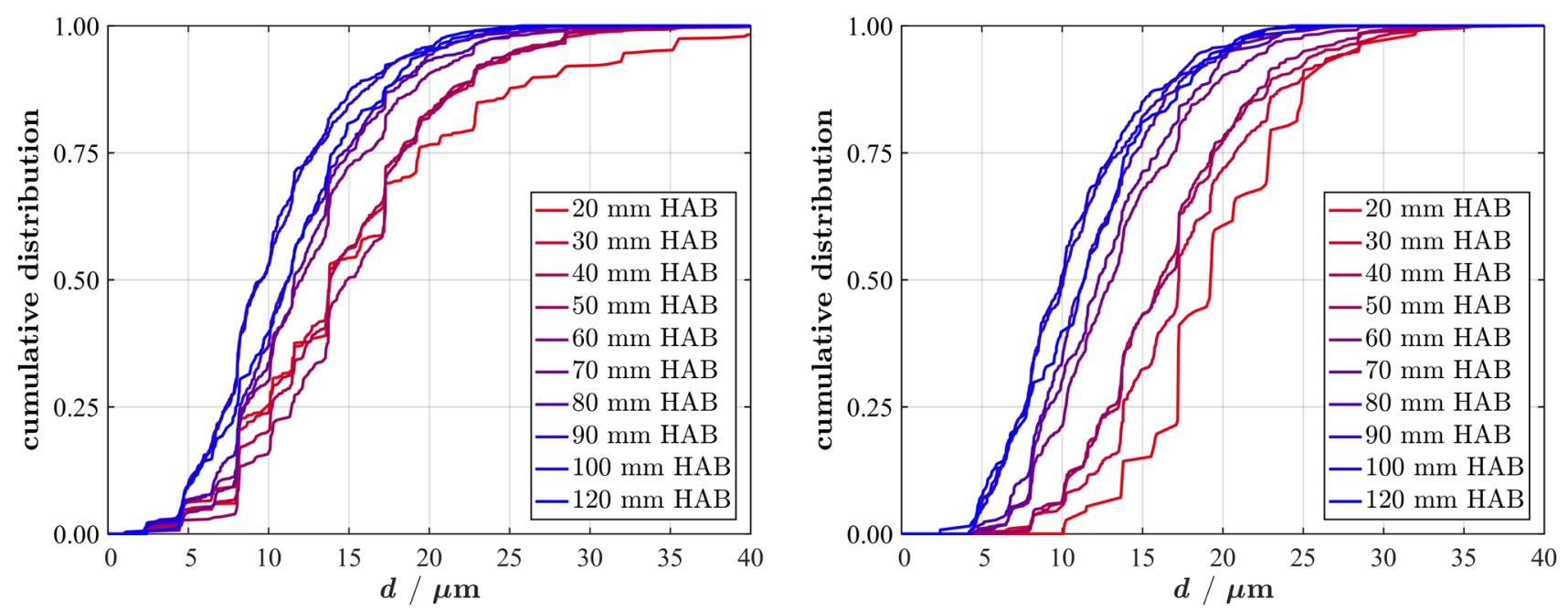

Fig. 6 Comparison of cumulative distributions of ethanol droplets along investigated HAB derived from automated pre-filtering (left) and after manual verification (right); coflow gas: air, $f$-number of camera: $f / 2.0$

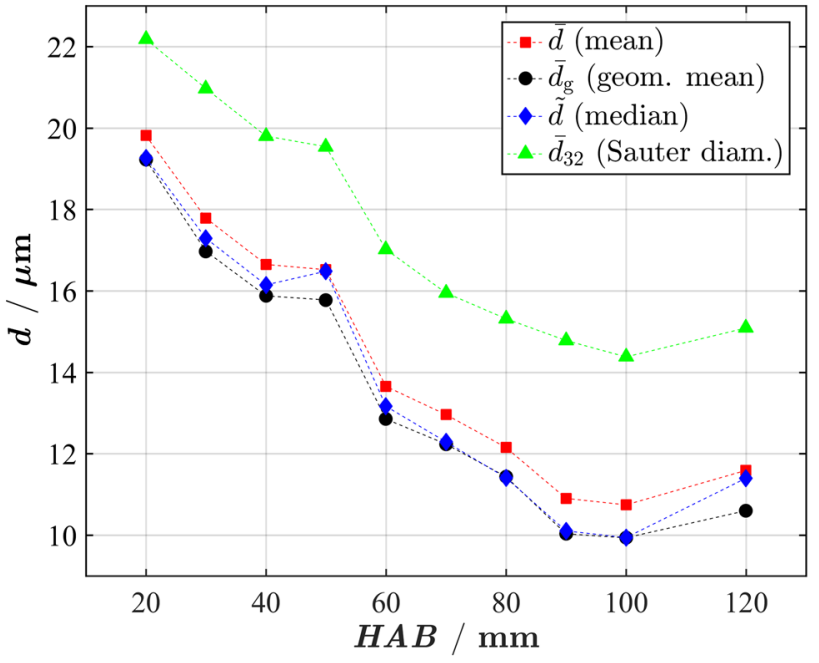

Fig. 7 Comparison of different mean values for ethanol droplets sizes, coflow gas: air, $f$-number of camera: $f / 2.0$ (left); comparison of median values for ethanol droplets for nitrogen and air as coflow

amount of scattering data in the images is maximized due to the size of the measurement volume. Decreasing the size of the aperture (and thus increasing the $f$-number) leads to a decreased size of the measurement volume as the acceptance region for scattered rays is decreased (see also Fig. 3, illustration a and c). The relative amount of images containing droplet data after a manual verification is reduced from $34.7 \%$ to $10.9 \%$ for $f / 4.0$ (medium-sized blue diamonds) and even down to $8.4 \%$ for $f / 5.6$ (small blue diamonds) at $40 \mathrm{~mm}$ HAB. At 20 mm HAB only $5.5 \%$ of the images are evaluable after manual verification using an $f / 2.0$ aperture. A reduction of the aperture size to an $f$-number of $f / 4.0$ results in a

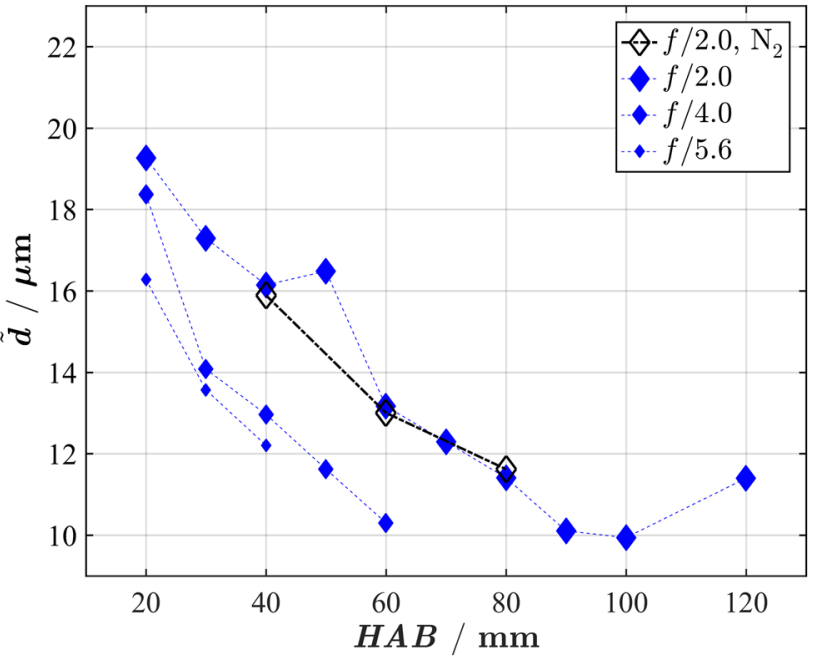

gases and for different $f$-numbers of the camera (right); all values derived from distributions after manual verification

considerably larger fraction of evaluable images (34.9\%). The reduction of the measurement volume leads to fewer superimposed scattering data and thus to a reliable determination of droplet sizes. However, a further reduction of the $f$-number to $f / 5.6$ results in a reduction of the fraction of evaluable images down to $18.7 \%$. Although the general trend of a decreasing mean droplet size persist for all $f$-numbers, the absolute values differ. With decreasing $f$-number (increasing the size of the observed measurement volume), the median value increases for all heights. This can be explained by the droplet size distribution in the spray which is depending on its radial position [25]: for the large 
measurement volume (f/2.0), not only droplets on the central spray axis are measured, but also droplets from the outer region of the spray. Following the results of Bieber et al. [25], the droplet size increases towards the edges in the spray of the SpraySyn burner. Reducing the size of the aperture leads to a more central measurement volume, where smaller droplets are present on average. However, although having a better spatial resolution for smaller $f$-numbers, the datarate also drastically decreases leading to almost no droplet data at larger heights.

Table 2 compares the arithmetic mean values and Sauter mean diameters derived from the PDA measurements conducted by Bieber et al. [25] to those obtained from WALS measurements after manual verification. Data were recorded at $\mathrm{HAB}=30 \mathrm{~mm}$ with both techniques (PDA and WALS) on different SpraySyn burners, identical in construction. PDA data are taken from the centerline of the burner and compared to WALS data acquired with an aperture size of the camera of $f / 5.6$ to limit the comparison to the relevant central region. The Sauter mean diameter determined with the WALS technique shows very good agreement with that from PDA measurements, the deviation is less than $8 \%$. However,

Table 2 Comparison of mean values and Sauter mean diameters of ethanol droplets at $\mathrm{HAB}=30 \mathrm{~mm}$ derived from WALS measurements (coflow gas: air, $f$-number of camera: $f / 5.6)$ after manual verification and from PDA-measurements [25]; relative deviation to PDA data in brackets

\begin{tabular}{lll}
\hline Technique & $-\bar{d} / \mu \mathrm{m}$ & $d_{32} / \mu \mathrm{m}$ \\
\hline PDA & 8.4 & 15.9 \\
WALS & $12.6(+50.2 \%)$ & $17.2(+7.9 \%)$ \\
\hline
\end{tabular}

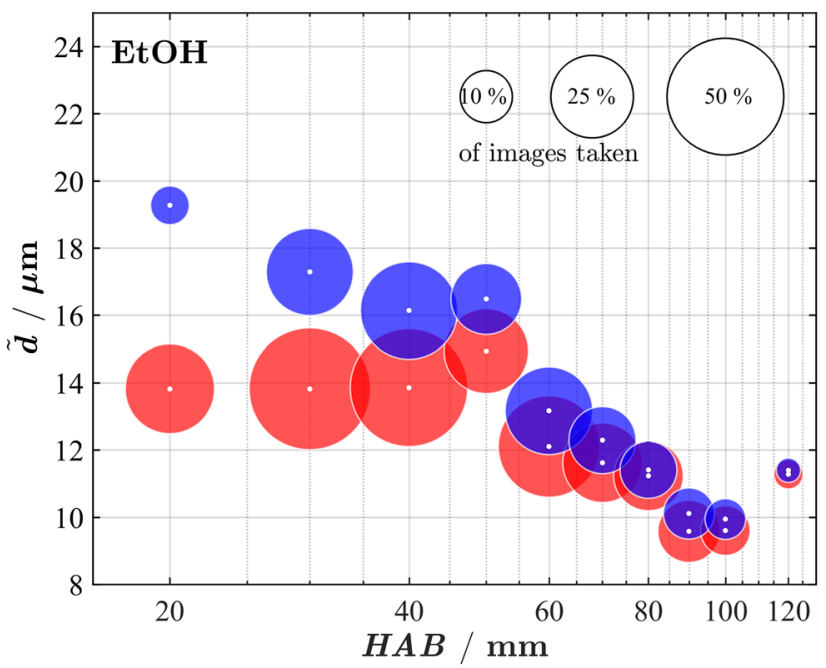

Fig. 8 Comparison of median values for ethanol (EtOH, left) and mixture of EtOH and EHA (right) from computer-based evaluation (red circles) and after manual verification (blue circles), circle size the arithmetic mean shows a rather high deviation of around $50 \%$. This indicates that the distribution derived from PDA data contains much smaller droplets than that obtained from WALS, which significantly reduces the arithmetic mean. Although a reduction of the WALS measurement volume minimizes the probability that superimposed scattered light of several single droplets will be detected, it cannot be totally avoided, especially towards smaller HABs with increasing spray density. If multiple scattering from one large droplet and several smaller droplets is detected simultaneously, the intensity of the large droplet clearly dominates the superimposed scattering signal and thus only the large droplet is identified in the evaluation routine. Probably, a significant amount of small droplets is rejected leading to a higher mean value compared to PDA, yet this has a negligible effect on the Sauter mean diameter. Approaching smaller HABs mean droplet sizes derived from PDA data are further decreasing [25]. Our data show an opposite tendency, which is caused by an increasingly difficult recognition of small droplets due to their low signal intensity and a superposition of scattered light from several droplets.

Figure 8 shows the median values for both solvents, pure ethanol (left figure) and the mixture of ethanol and EHA (right figure) derived from computer-based evaluation (red circles) and after manual verification (blue circles). The circle size corresponds to the relative number of evaluable images of all images taken at a specific observation point. For both solvents, the fraction of evaluable images after a manual verification is maximized at $40 \mathrm{~mm} \mathrm{HAB}$ and decreases towards both smaller and larger HAB. It ranges between $2.2 \%$ and $34.7 \%$ for the ethanol spray and between $3.1 \%$ and $49.3 \%$ for the mixture of ethanol and

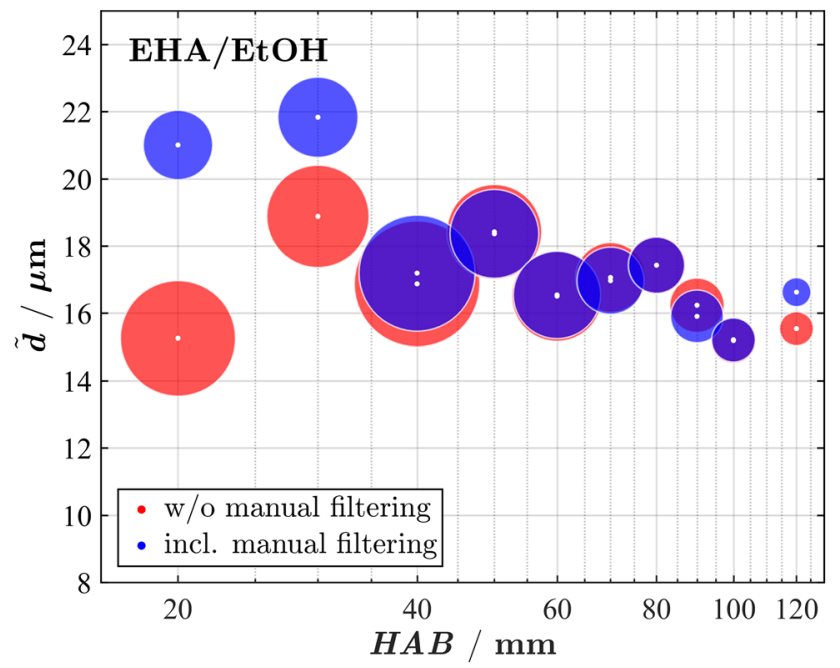

corresponds to percentage of evaluable images based on total images taken at one $\mathrm{HAB}$; coflow gas: air, $f$-number of camera: $f / 2.0$ 
EHA. Towards smaller HABs more superimposed scattering data from multiple droplets are detected that cannot be evaluated correctly by the computer-based evaluation routine used in this work. Especially at smaller HABs and larger deviations of the median of the derived droplet distribution the amount of evaluable images is significantly reduced between computer-based evaluation and manual verification, e.g., at $20 \mathrm{~mm} \mathrm{HAB}$ evaluable images for the ethanol spray are reduced by more than $80 \%$ from 584 to 110 images out of a total of 1995 images taken. Towards larger HABs the spray density and thus the probability of detecting scattering signals at all decreases.

For ethanol, the deviation between the median values derived from computer-based evaluation and manual verification is relatively high, especially at smaller HABs up to $50 \mathrm{~mm}$. The maximum deviation at $20 \mathrm{~mm} \mathrm{HAB}$ is $39.5 \%$ with a decreasing tendency towards $10.3 \%$ at $50 \mathrm{~mm}$ HAB. At larger heights, the deviation is less than $10 \%$ with a further decreasing tendency. A similar trend can be observed for the mixture of ethanol and EHA, but with quite smaller deviations. Here, the deviation is $37.6 \%$ at $20 \mathrm{~mm} \mathrm{HAB}$ and $15.6 \%$ at $30 \mathrm{~mm} \mathrm{HAB}$. Larger heights between 40 and $100 \mathrm{~mm}$ HAB exhibit deviations of less than $2 \%$, at $120 \mathrm{~mm}$ the deviation increases slightly again. The median values for pure ethanol and the mixture with EHA are both decreasing with increasing $\mathrm{HAB}$, yet the droplets in the mixture are generally larger with a range of 15.2-21.8 $\mu \mathrm{m}$. For both solvents, the volume flow is kept constant, yet the median value for droplets of the ethanol spray is smaller by between $6 \%$ and $37 \%$ - depending on the $\mathrm{HAB}$ - than for the mixture of ethanol and EHA. This indicates that the atomization of the ethanol spray is much better leading to generally smaller droplets with a higher number density in the spray along with the whole axial extent of the spray flame. Thus, the probability of detecting superimposed scattering from multiple droplets is increased for the ethanol spray leading to higher deviations between computer-based evaluation and manual verification at all HABs.

For a robust evaluation of droplet sizes from Mie scattering on the basis of a database, assumptions were made (see Sect. 4) that may lead to uncertainties in the droplet sizes determined. The mixture of ethanol and EHA, two substances with a large difference in their individual boiling points $\left(\Delta T_{\text {boil }} \sim 149 \mathrm{~K}[50]\right)$ might result in an increased evaporation rate of the component with lower boiling point (here: ethanol) and thus a change in the average refractive index of the droplet with increasing HAB. We estimated the possible deviation of an over- and underestimation of the refractive index by re-evaluating the scattering data taken at $60 \mathrm{~mm} \mathrm{HAB}$ with the mixture of ethanol and EHA using different refractive indices for the database. The obtained median values ( $16.5 \mu \mathrm{m}$ for both computer-based and manual verification) and amount of evaluable images (29.9\% for computer-based evaluation, $28.0 \%$ for manual verification) at this observation point show very good agreement between both evaluations (see Fig. 8, right). Thus, in the following only results from the computer-based evaluation are compared. With the refractive index of pure EHA (1.425), reflecting an overestimation in the refractive index by $1.4 \%$, the median shifts towards $16.7 \mu \mathrm{m}$; an underestimation in the refractive index by $-2.9 \%$ (assuming pure ethanol as solvent) results in a slightly lower value of $16.0 \mu \mathrm{m}$. Both deviations are less than $2 \%$ and thus negligible especially when considering that extreme values for the composition have been used for this estimate. Similarly, the effect of a temperature change on the refractive index can be safely neglected, for example, the refractive index of ethanol changes by roughly 0.01 for a temperature variation of $20 \mathrm{~K}$ [52].

As the shape of the droplets may differ from perfect spheres, deviations between the real equivalent droplet size and the obtained sphere size may occur. Therefore, we applied our evaluation routine to simulated scattering data from spheroids using the Matlab-based $T$-matrix method of Somerville et al. [53] to quantify these deviations. Example calculations were carried out for spheroids with equivalent spherical diameters (ESD) of up to $9 \mu \mathrm{m}$ corresponding to a size value of up to $x \approx 50$, which is the limit of the $T$-matrix simulations [53]. We derived scattering data for spheroid sizes between $2 \mu \mathrm{m}$ and $9 \mu \mathrm{m}$ ESD and altered the orientation of the spheroid along the three main axes of threedimensional space with respect to the direction of incident laser light. Besides, we varied the ratios of both semi-axes between 0.9 and 1.1 representing typical shape oscillations for droplets in this size range [54]. The obtained average overestimation of $0.18 \mu \mathrm{m}$ with an average relative deviation of $3.1 \%$ between the sphere diameters derived from our evaluation and the ESD of the spheroids is relatively small. As the relative deviations are quite constant for the observed ESDs, we assume a similar tendency for droplets of sizes exceeding the calculation limit of $9 \mu \mathrm{m}$ ESD. The assumption of spherical droplets is thus a good approximation for which deviations in the recovered size distribution can be neglected.

A dislocation of the droplet from the center of the measurement volume not only causes a signal cutoff but also a deviation between the detection angle of the measurement system and the angle of the scattered light and is also dependent on the size of the camera aperture (c.f. Figure 3, $\mathrm{b}$ and c). Under $90^{\circ}$ scattering angle the size of the observed measurement volume is minimized, yet the angular deviation of a dislocated droplet is maximized. At smaller scattering angles the angular deviation is generally reduced, however, the possible dislocation of droplets is increased due to the increased size of the observed measurement volume. The maximum possible angular deviation is in the end almost 
constant for all scattering angles as those competitive effects cancel out. In our case, for $f$-numbers of $f / 4.0$ and $f / 5.6$ the maximum angular deviation at all scattering angles is less than $0.2^{\circ}$ and thus cannot be resolved by the measurement system. For an $f / 2.0$-aperture this maximum angular shift is about $0.3^{\circ}$, which is slightly higher than the resolution of the experiment of $0.2^{\circ}$. As a result, significant deviations in the determined droplet sizes are only expected for large droplets (approx. $>60 \mu \mathrm{m}$ ) for which the scattering signal oscillation shows very high frequencies.

\section{Conclusion}

The wide-angle light scattering (WALS) technique was successfully used for the determination of droplet sizes and their distributions in a standardized burner for spray flame synthesis (SFS) of nanoparticles (SpraySyn burner). Measurements were conducted on sprays of ethanol and a mixture of ethanol and 2-ethylhexanoic acid (EHA) without any precursors to inhibit nanoparticle formation. A pulsed laser in combination with a CCD-camera was used to acquire singleshot scattering images. Droplet sizes were derived from each scattering data set by a computer-based evaluation routine conducting a least square minimization of residuals between measured and calculated scattering data. For the latter one, a database on the basis of the Mie theory was generated assuming spherical droplets with a constant refractive index. All data fits from computer-based evaluation were verified manually and their results were compared. Droplet sizes and their distributions were determined along the center axis of the spray at heights above burner (HAB) between 20 and $120 \mathrm{~mm}$. We also examined the influence of the size of the measurement volume and the presence of oxygen in the coflow of the spray flame on the droplet size distribution.

From our results, we can conclude that for both solvents the droplet sizes are log-normally distributed and decreasing with increasing $\mathrm{HAB}$ due to evaporation in the hot flame. Due to different boiling temperatures, droplets containing EHA are generally larger and evaporate more slowly. Furthermore, the use of nitrogen instead of air as coflow gas does not influence the droplet formation and evaporation significantly, yet, the influence of the coflow gas on the particle morphology produced by the SFS still has to be investigated. Especially at smaller HABs the higher droplet density results in a superposition of scattering from multiple droplets. Pure computer-based evaluation in these heights leads to an underestimation of droplet sizes requiring a manual verification. However, at larger HABs these deviations are minimized.

Regarding the size of the measurement volume, we can conclude: at smaller HABs a reduced measurement volume should be applied as scattering from multiple droplets leads to errors in the evaluation. However, at larger HABs it should be increased to increase the droplet data rate in the images. As the size of the measurement volume can be adjusted by simply changing the size of the aperture of the camera lens, an adaption to varying process conditions is straightforward with the WALS technique.

As the need for manual post-filtering for some process conditions is currently a major limitation for routine application of the technique, more sophisticated filters have to be developed. To this end, we will have a closer look at the manual post-processing and try to transfer the criteria applied by a human operator into computer-based routines. In this context, the use of artificial neural networks might be a particularly promising approach. In future work, we also want to extend the algorithms to the evaluation of scattering data from multiple droplets and the superposition of scattering from droplets with the aim to use WALS for both droplet and particle sizing within a single process, which will help to better understand the chain of effects in the SFS process.

Acknowledgements Open Access funding provided by Projekt DEAL. The authors gratefully acknowledge funding by the German Research Foundation (DFG) under Grant No. WI1602/15-1 within the priority program "Nanoparticle Synthesis in Spray Flames" SPP 1980.

Open Access This article is licensed under a Creative Commons Attribution 4.0 International License, which permits use, sharing, adaptation, distribution and reproduction in any medium or format, as long as you give appropriate credit to the original author(s) and the source, provide a link to the Creative Commons licence, and indicate if changes were made. The images or other third party material in this article are included in the article's Creative Commons licence, unless indicated otherwise in a credit line to the material. If material is not included in the article's Creative Commons licence and your intended use is not permitted by statutory regulation or exceeds the permitted use, you will need to obtain permission directly from the copyright holder. To view a copy of this licence, visit http://creativecommons.org/licenses/by/4.0/.

\section{References}

1. J.B. Donnet, Carbon black: science and technology, 2nd edn (Marcel Dekker Inc, New York, 1993)

2. A. Lorke, M. Winterer, R. Schmeckel, C. Schulz, Nanoparticles from the gasphase (Springer-Verlag, Berlin, Heidelberg, 2012)

3. G.W. Kriechbaum, P. Kleinschmit, Angew Chem Int Ed Engl 28, 1416 (1989)

4. G.L. Messing, S.-C. Zhang, G.V. Jayanthi, J Am Ceram Soc 76, 2707 (1993)

5. A. Gurav, T. Kodas, T. Pluym, Y. Xiong, Aerosol Sci Technol 19, 411 (1993)

6. R. Mueller, L. Mädler, S.E. Pratsinis, Chem Eng Sci 58, 1969 (2003)

7. K. Okuyama, I. Wuled-Lenggoro, Chem Eng Sci 58, 537 (2003)

8. R. Strobel, S.E. Pratsinis, J Mater Chem 17, 4743 (2007)

9. D. Kilian, S. Engel, B. Borsdorf, Y. Gao, A.F. Kögler, S. Kobler, T. Seeger, S. Will, A. Leipertz, W. Peukert, J Aerosol Sci 69, 82 (2014) 
10. C. Schulz, T. Dreier, M. Fikri, H. Wiggers, Proc Combust Inst 37, 83 (2019)

11. S. Li, Y. Ren, P. Biswas, S.D. Tse, Prog Energy Combust Sci 55, 1 (2016)

12. K. Wegner, S.E. Pratsinis, Chem Eng Sci 58, 4581 (2003)

13. J. Menser, S. Kluge, H. Wiggers, T. Dreier, C. Schulz, in Approach to standardize a spray-flame nanoparticle synthesis burner. In: Proceedings of the European Combustion Meeting, Budapest, Hungary (2015)

14. F. Schneider, S. Suleiman, J. Menser, E. Borukhovich, I. Wlokas, A. Kempf, H. Wiggers, C. Schulz, Rev Sci Instrum 90, 085108 (2019)

15. F. Durst, A. Melling, J.H. Whitelaw, STIA 76, 47019 (1976)

16. L.E. Drain, The laser doppler technique (Wiley, United Kingdom, 1980)

17. C. Tropea, T.-H. Xu, F. Onofri, G. Géhan, P. Haugen, M. Stieglmeier, Part Part Syst Char 13, 165 (1996)

18. C.R. Tuck, M.C.B. Ellis, P.C.H. Miller, Crop Prot 16, 619 (1997)

19. X. Liu, W.H. Doub, C. Guo, Int J Pharm 388, 82 (2010)

20. H.D.V. Stein, H.J. Pfeifer, Metro 5, 59 (1969)

21. K. Bauckhage, Part Part Syst Char 5, 16 (1988)

22. K. Bauckhage, H.-H. Floegel, U. Fritsching, R. Hiller, Part Part Syst Char 5, 66 (1988)

23. H.-E. Albrecht, M. Borys, M. Wenzel, T. Wriedt, Part Part Syst Char 11, 339 (1994)

24. H.-E. Albrecht, M. Wenzel, M. Borys, Part Part Syst Char 13, 18 (1996)

25. M. Bieber, R. Tischendorf, H.-J. Schmid, M. Reddemann, R. Kneer, in Influence of precursor concentration on spray and particle formation in flame spray pyrolysis. ILASS Europe 2019, 29th Conference on Liquid Atomization and Spray Systems, Paris, France (2019). Paris, France

26. M.F.B. Stodt, J. Kiefer, U. Fritsching, Exp Fluids 60, 125 (2019)

27. C.M. Sorensen, Aerosol Sci Technol 35, 648 (2001)

28. J. Reimann, S.A. Kuhlmann, S. Will, Appl Phys B 96, 583 (2009)

29. M. Altenhoff, S. Aßmann, J.F.A. Perlitz, F.J.T. Huber, S. Will, Appl Phys B 125, 176 (2019)

30. H. Oltmann, J. Reimann, S. Will, Combust Flame 157, 516 (2010)

31. H. Oltmann, J. Reimann, S. Will, Appl Phys B 106, 171 (2012)

32. F.J.T. Huber, S. Will, J Aerosol Sci 119, 62 (2018)

33. F.J.T. Huber, S. Will, K.J. Daun, J Quant Spectros Radiat Transfer 184, 27 (2016)

34. G. Mie, Ann Phys 330, 377 (1908)
35. H.C. Hulst, Light scattering by small particles (Wiley, New York, 1957)

36. C.F. Bohren, D.R. Hufmann, Absorption and scattering by a sphere (Wiley, Weinheim, 1998)

37. M. Kerker, The scattering of light and other electromagnetic radiation (Academic Press, New York, 1969)

38. W.J. Wiscombe, Mie scattering calculations: advances in technique and fast, vector-speed computer codes (National Center for Atmospheric Research, Boulder, 1979)

39. F.J.T. Huber, M. Altenhoff, S. Will, Rev Sci Instrum 87, 053102 (2016)

40. G.W. Kattawar, G.N. Plass, Appl Opt 6, 1377 (1967)

41. J.V. Dave, IBM J Res Dev 13, 302 (1969)

42. P. Latimer, J Colloid Interface Sci 53, 102 (1975)

43. V.E. Cachorro, L.L. Salcedo, JEWA 5, 913 (1991)

44. Z.S. Wu, L.X. Guo, K.F. Ren, G. Gouesbet, G. Gréhan, Appl Opt 36, 5188 (1997)

45. I.W. Sudiarta, P. Chylek, J Opt Soc Am A 18, 1275 (2001)

46. H. Du, Appl Opt 43, 1951 (2004)

47. M. Retsch, M. Schmelzeisen, H.-J. Butt, E.L. Thomas, Nano Lett 11, 1389 (2011)

48. Z. Wang, N. An, F. Shen, H. Zhou, Y. Sun, Z. Jiang, Y. Han, Y. Li, Z. Guo, Nanoscale Res Lett 12, 58 (2017)

49. J. Rheims, J. Köser, T. Wriedt, Meas Sci Technol 8, 601 (1997)

50. J.R. Rumble, CRC handbook of chemistry and physics 100th edition (Internet Version 2019) (CRC Press/Taylor \& Francis, Boca Raton, 2019)

51. F. Huber, Entwicklung eines Systems zur Online-Charakterisierung von Nanopartikeln in der Gasphase auf Basis der Weitwinkel-Lichtstreuung und der Laserinduzierten Inkandeszenz (Shaker Verlag, Aachen, 2017)

52. R.J. Riobóo, M. Philipp, M. Ramos, J.-K. Krüger, Eur Phys J E 30, 19 (2009)

53. W.R.C. Somerville, B. Auguié, E.C. Le Ru, J Quant Spectros Radiat Transfer 174, 39 (2016)

54. R.E.H. Miles, M.W.J. Glerum, H.C. Boyer, J.S. Walker, C.S. Dutcher, B.R. Bzdek, J Phys Chem A 123, 3021 (2019)

Publisher's Note Springer Nature remains neutral with regard to jurisdictional claims in published maps and institutional affiliations. 